\title{
Depresión posparto en padres ¿por qué también es importante?
}

\author{
Paternal postpartum depression. Why is it also important?
}

\author{
Francisca Pérez C. ${ }^{a}$, Paulina Brahm M. ${ }^{\text {b }}$
}

aFacultad de Psicología, Universidad Alberto Hurtado, Santiago

bepartamento de Medicina Familiar, Pontificia Universidad Católica de Chile, Santiago

Recibido el 14 de junio de 2017; aceptado el 31 de julio de 2017

\section{Resumen}

La supervisión de salud es una oportunidad privilegiada para acompañar a las nuevas familias en su transición a la parentalidad. Este período trae importantes desafíos para la madre y el padre, y en ocasiones la demanda puede superar los recursos emocionales de la familia; este desbalance puede favorecer que madres y padres presenten sintomatología depresiva. La depresión posparto puede afectar de uno a dos de cada diez hombres, siendo la depresión materna un factor de riesgo importante para desarrollarla. La depresión posparto en el padre impacta a todos los miembros de la familia, siendo el desarrollo infantil, el vínculo y la salud mental del niño los que pueden verse alteradas a corto, mediano y largo plazo. Parece relevante por lo tanto pensar en un tamizaje para pesquisar precozmente la depresión posparto no sólo en madres sino también en padres y dar así un primer paso a ampliar la mirada desde la diada madre-hijo a la triada. El control de salud del niño es una oportunidad única para poder realizar esta pesquisa, sin embargo la validación de una escala de tamizaje de depresión posparto en padres chilenos es una tarea pendiente.

\section{Palabras clave:}

Depresión posparto paterna, evaluación, Escala de Depresión Posparto de Edimburgo

\begin{abstract}
Health supervision is a privileged opportunity to walk along with the new families during their transition to parenthood. This period is challenging for both mother and father, and there is the potential danger that demand surpasses the existing resources of the family. This imbalance may lead to mothers and fathers to develop depressive symptomatology. Postpartum depression may affect one to two out of ten men, and maternal depression is a major risk factor for developing it. The postpartum depression in the father impact all the family members, being the child development, the bonding, and the child's mental health which can be disturb at a short, medium and long term. Therefore, it seems to be relevant to think about screening for post partum depression not only in mothers but also fathers, and give a first step to broaden the gaze from the dyad to the triad. The health supervision is a unique opportunity to be able to carry out this screening; however, the validation of a postpartum screening test for Chilean fathers is a pending task.
\end{abstract}

\section{Keywords:} Paternal postpartum depression, assessment, Edinburgh Postnatal Depression Scale 


\section{Introducción}

Durante la supervisión de salud del niño*, el médico tiene la oportunidad privilegiada de acompañar a la nueva familia en su transición hacia la parentalidad, etapa comprendida por el embarazo, parto y posparto. Frecuentemente es un período de alegría y expectación, pero también implica una serie ajustes y desafíos para la pareja y la familia emergente ${ }^{1}$. Esta etapa se ha definido como una crisis normativa en la vida de las personas $^{2}$, ya que los nuevos padres deben balancear no sólo las demandas de su vida personal, laboral, social, sino también las presiones económicas y las necesidades emocionales de la familia, todo esto dentro del desconocido rol paternal haciendo frente a los requerimientos de la coparentalidad y de la coordinación de los padres ${ }^{3,4}$. Por lo tanto, si bien existe la posibilidad de seguir creciendo y desarrollándose como individuos y como familia, también existe el peligro potencial de que la demanda sobrepase los recursos existentes y que aumenten por lo tanto significativamente los niveles de estrés, ansiedad y sintomatología depresiva en ambos miembros de la pareja, lo que incide negativamente a nivel personal, familiar y en el desarrollo de los niños ${ }^{5-7}$. Son por lo tanto objetivos de este artículo exponer la relevancia de la salud mental -en particular de la depresión posparto- en el padre durante este período, conocer el impacto de ésta en el niño y su familia; y plantear el rol del médico en el diagnóstico precoz de esta enfermedad.

\section{Cambios biopsicosociales de la nueva familia e impacto en la salud mental de la madre y el padre}

Históricamente, la llegada de un hijo se ha asociado a los cambios biológicos, psicológicos y sociales por los que atraviesa la mujer, dado que es ella quien vive en primera persona el embarazo, el parto y la lactancia. Está descrito que muchas mujeres, después del parto, son muy sensibles a los cambios hormonales que experimenta su cuerpo, lo que tiene un correlato anímico que se presenta con tristeza las dos primeras semanas posparto, fenómeno conocido como baby blues, que en general remite espontáneamente. Sin embargo, cifras internacionales reportan que hasta un $20 \%$ experimentará sintomatología depresiva durante más de un $\mathrm{mes}^{8}$. En Chile, hay series que reportan alrededor de un $14 \%$ de screening positivo para depresión posparto (DPP) en madres ${ }^{9}$, sin embargo se ha observado una distribución desigual de acuerdo al nivel socioeconómico, con una prevalencia de un $41,3 \%$ en sectores bajos versus un $27,7 \%$ en sectores altos ${ }^{10}$.

Si bien el hombre no experimenta los cambios fisiológicos de la gestación y el puerperio - e incluso puede no haberse enterado que será padre - hoy se sabe que también atraviesa por profundos cambios psicológicos, principalmente en relación a su nuevo rol y sus implicancias ${ }^{1,11-12}$. De esta forma, los padres pueden presentar sintomatología depresiva en el posparto, habiendo sido descrita una prevalencia de $10,4 \%$ a nivel mundial ${ }^{5}$. En una revisión de la literatura, se encontraron rangos muy dispares de prevalencia: de entre 1,8\% a $47 \%$, en Turquía y Suecia respectivamente. Estas diferencias tendrían que ver principalmente con las metodologías de los estudios, el tiempo de evaluación y las características de la población estudiada ${ }^{13}$. Si se observa el grupo de padres cuyas parejas presentan DPP, uno cada dos a uno de cada cuatro hombres desarrollará depresión también en este período ${ }^{5}$. En Chile no contamos con datos epidemiológicos del fenómeno, pero un estudio exploratorio evaluó a población masculina de clase media baja dos meses posparto con la Escala de Depresión Posparto de Edimburgo, mostró una frecuencia de un $18,5 \%$ tomando como puntaje de corte 10 puntos $^{14}$. Por otra parte, si bien existe una considerable variabilidad en el período de tiempo en que los padres presentan DPP, el período entre los tres y seis meses posparto muestra la incidencia más alta ${ }^{5}$.

Entre de los factores de riesgo para desarrollar DPP en hombres se han identificado un historial personal de depresión, una relación de pareja conflictiva, la falta de apoyo de la familia y los amigos, el desempleo, una mayor edad y un menor nivel educacional, entre otros. Sin embargo la presencia de sintomatología depresiva materna está descrita como una de las asociaciones más robustas ${ }^{7,15,16}$.

\section{Impacto de la depresión posparto paterna}

La DPP paterna se ha asociado a variadas condiciones futuras, afectando el funcionamiento familiar, el bienestar de los integrantes de la familia, la satisfacción marital y la economía de países industrializados ${ }^{17-20}$.

Asimismo, diversos estudios han reportado como la sintomatología depresiva paterna en el posparto impacta el desarrollo infantil. En el corto plazo hay evidencia que padres con sintomatología depresiva perciben a sus hijos con temperamento más difícil que padres sin sintomatología ${ }^{21}$, pueden favorecer episo-

\footnotetext{
* Los sustantivos masculinos como "niño" tanto en singular como en plural se utilizaron en el texto como genéricos, en otras palabras incluyen a toda la población sin distinción de sexo. Fuente: http://www.rae.es/consultas/los-ciudadanos-y-las-ciudadanas-los-ninos-y-las-ninas.
} 
dios de llanto prolongado y dificultades en el sueño de sus hijos pequeños ${ }^{6}$, existen mayores dificultades en la interacción ${ }^{22-24}$, los lactantes presentarían mayores dificultades en la conducta alimentaria ${ }^{19} \mathrm{y}$ además existe un mayor riesgo de desarrollar un estilo de apego inseguro ${ }^{25}$. En el mediano plazo, los hijos de padres con DPP tienden a presentar un aumento tanto en la frecuencia de alteraciones del comportamiento, tales como problemas de conducta o hiperactividad ${ }^{25}$, como en los problemas emocionales ${ }^{26}$. Estos altos niveles de problemas emocionales y del comportamiento se han observado a la edad de tres años y medio, especialmente en hijos varones ${ }^{6}$. Al largo plazo, se evidencia mayor riesgo de presentar un bajo funcionamiento psicosocial y un aumento de riesgo de la ideación e intento suicidas en sus hijos durante la adultez temprana, y un aumento de depresión en sus hijas durante este mismo período ${ }^{25}$.

\section{Tamizaje}

A pesar que la Organización Mundial de la Salud ha recomendado evaluar la salud mental perinatal sólo de la madre y no de ambos padres ${ }^{27}$, y que en Chile se ha descrito una baja cobertura de tamizaje de DPP materna en el sistema privado y barreras en el acceso al tratamiento en el sistema público ${ }^{9,28}$, dada la relevancia del problema, parece importante reflexionar respecto a la necesidad de implementar en nuestro país un tamizaje para la DPP también en padres.

En la literatura internacional, la mayoría de los estudios publicados hasta le fecha han utilizado como principal instrumento la Escala de Edimburgo para Depresión Posparto (EDPE), la cual ha sido validada sólo en algunos países como Australia y Portugal para población masculina ${ }^{29,30}$. Además de existir entonces la limitación de la validez de los resultados, existen estudios cualitativos que señalan que la sintomatología depresiva masculina tendría ciertas especificidades, las que no son pesquisadas por este instrumento. Algunas de estas manifestaciones son hostilidad, conflicto, enojo ${ }^{31} y$ conductas evitativas como incrementar la jornada laboral, el deporte, la promiscuidad sexual, y el consumo de sustancias ${ }^{32}$.

\section{Rol del médico en la supervisión de salud}

Resulta entonces pertinente ampliar la mirada diádica madre-hijo y considerar también al padre en los controles perinatales de salud, entendiendo así que la salud mental no existe en forma aislada, sino que es fundamentalmente un fenómeno contextual y relacio- nal. En este contexto, los pediatras y médicos familiares que ven niños tienen un rol fundamental en reconocer la importancia de incorporar a los padres como figuras relevantes en la salud y desarrollo de sus hijos ${ }^{33}$.

Dentro de las oportunidades de involucrar al padre dentro de la atención de salud están: mostrar aprecio por ir al control de salud, hablarle tanto al padre como a la madre, mostrar como los niños ven a sus padres como modelos de comportamiento, educar a los padres sobre como apoyar a la madre en la lactancia, dar énfasis en el rol único que juega cada uno de los padres en la crianza, entre otros ${ }^{33}$. De esta forma, la mirada sistémica se vuelve fundamental a la hora de evaluar e intervenir -fundamentalmente en las primeras etapas del niño-ya que durante el primer año de vida del lactante se sientan las bases de su salud mental futura. El diagnóstico precoz y la intervención oportuna no sólo de la DPP materna, sino que también paterna, independiente del tipo de relación que exista entre la pareja parental, es clave para fomentar el ejercicio de una parentalidad responsable y el bienestar familiar.

\section{Actuales desafíos}

Sin duda es necesario seguir estudiando el fenómeno de la DPP paterna, en un primer momento a través de estudios exploratorios que evalúen la vivencia de los síntomas depresivos en padres, para poder luego validar en Chile alguna escala de tamizaje breve y con buena aceptación para ser aplicada en nuestra población. De esta forma los profesionales de la salud a cargo de los controles pre y postnatales podrían incorporar dentro de éstos la pesquisa en DPP tanto de la madre como del padre. Se sugiere que la primera pesquisa se realice durante el embarazo, considerando que tanto en mujeres como en hombres la depresión durante el embarazo suele mantenerse en el posparto, luego al segundo y sexto mes del lactante, y además cuando exista sospecha de sintomatología depresiva o alteración del vínculo. Por último, considerando las barreras que existen en Chile en el acceso a tratamiento de las mujeres que han sido diagnosticadas con $\mathrm{DPP}^{28}$, es un desafío habilitar al sistema para poder dar cobertura, gestionar y monitorear las derivaciones y tratamientos tanto de madres como de padres, de manera de poder disminuir el riesgo y las consecuencias de la DPP para los padres y sus hijos.

\section{Conflicto de intereses}

Los autores declaran no tener conflicto de intereses. 


\section{Referencias}

1. Cierpka M, Scholtes K, Frey B, Köhler H. Der Übergang zur Elternschaft. In: H K, ed. Handbuch der Kleinkindforschung. Bern: Huber; 2011.

2. Frevert G, Cierpka M, Joraschky P. Familiäre Lebenszyklen. In: Cierpka M, ed. Handbuch der Familiendiagnostik. 3 ed. Heidelberg: Springer; 2008.

3. McHale J. When infants grow up in multiperson relationship systems. Infant Ment Health J. 2007;28(4):370-92.

4. Genesoni L, Tallandini MA. Men's psychological transition to fatherhood: an analysis of the literature, 1989-2008. Birth. 2009;36(4):305-18.

5. Paulson J, Bazemore S. Prenatal and Postpartum Depression in Fathers and Its Association With Maternal Depression A Meta-analysis. JAMA. 2010;303(10).

6. Baldwin S, Kelly P. Postnatal Depression: Don't reinvented the wheel. Community Practitioner. 2015;88(9):37-40.

7. Paulson J, Bazemore S, Goodman J, Leiferman J. The course and interrelationship of maternal and paternal perinatal depression. Archives of Women's Mental Health. 2016:1-9.

8. O'Hara M. Postpartum depression: what we know. J Clin Psychol 2009;65:1258-69.

9. Schonhaut Berman L, Podesta Lopez L. Rol del pediatra frente a la depresión posparto. Rev Chil Pediatr. 2016;87(1):13.

10. Jadresic E, Araya R, Jara C. Validation of the Edinburgh Postnatal Depression Scale (EPDS) in Chilean postpartum women. J Psychosom Obstet Gynaecol. 1995;16(4):187-91.

11. Cupa D, Riazuelo-Deschamps H. The paternal constellation: a pilot study in prenatal period. Sante Ment Que. 2001;26(1):58-78.

12. King V. Bedingungen der Elternschaftskonstellation Umgestaltungen der Identität von der Adoleszenz zu väterlichen und mütterlichen Kompetenzen. Kinderanalyse. 2010;18(1):1-27.

13. Pérez F, Brahm P, Riquelme S, Rivera C, Jaramillo K, Eickhorst A. Paternal postpartum depression: How has it been assessed? A literature review. Mental
Health \& Prevention. 2016;Available online 22 July 2017(In Press, Accepted Manuscript).

14. Pérez F, Catalán A, Morales A, Quinlan A, Riquelme D, Navarro J. Assessment of Postpartum Depression in a Group of Chilean Parents. Artículo no publicado: Universidad Alberto Hurtado; 2017.

15. Escriba-Agüir V, Artazcoz L. Gender differences in postpartum depression. A longitudinal cohort study. J Epidemiol Community Health. 2010;65(4).

16. Gao L, Chan S, Mao Q. Depression, perceived stress, and social support among first-time Chinese mothers and fathers in the postpartum period. Res Nurs Health. 2009;32(1):50-8.

17. Ramchandani P, Stein A, Evans J, O'Connor T, Team AS. Paternal depression in the postnatal period and child development: a prospective population study. Lancet. 2005;365(9478):2201-5.

18. Paulson J, Keefe H, Leiferman J. Early parental depression and child language development. J Child Psychol Psychiatry. 2009;50(3):254-62.

19. Cockshaw W, Muscat T, Obst P, Thorpe K. Paternal postnatal depressive symptoms, infant sleeping and feeding behaviors, and rigid parental regulation : a correlational study. Journal of Psychosomatic Obstetrics and Gynecology. 2014;35(4):124-131.

20. Ramchandani P, Psychogiou L, Vlachos H, et al. Paternal depression: an examination of its links with father, child and family functioning in the postnatal period. Depression and Anxiety. 2011;28:471-7.

21. Kerstis B, Engström G, Edlund B, Aarts C. Association between mothers' and fathers' depressive symptoms, sense of coherence and perception of their child's temperament in early parenthood in Sweden. Scandinavian Journal of Public Health. 2013;41:233-9.

22. Parfitt $Y$, Pikea A, Ayersb S. The impact of parents' mental health on parent-baby interaction: A prospective study Infant Behavior \& Development. 2013;36:599608.

23. Parfitt Y, Ayers S, Pike A, Jessop D,
Ford E. A prospective study of the parent-baby bond in men and women 15 months after birth. Journal of Reproductive and Infant Psychology. 2014;32(5):441-56.

24. Sethna V, Murray L, Netsi E, Psychogiou L, Ramchandani P. Paternal Depression in the Postnatal Period and Early Father-Infant Interactions. Parent Sci Pract. 2015;15:1-8.

25. Kim P, Swain JE. Sad Dads: Paternal Postpartum Depression. Psychiatry (Edgmont). 2007;4(2):35-47.

26. Butler L. Paternal Depression, Expressed Emotion and Child Emotional and Behavioral Problems. Exeter: Department of Clinical Psychology, University of Exeter; 2012.

27. WHO. Maternal and child mental health. 2016; http://www.who.int/ mental_health/maternal-child/en/, última visita 04.08.2016.

28. Rojas G, Santelices M, Martínez P, et al. Barreras de acceso a tratamiento de la depresión posparto en Centros de Atención Primaria de la Región Metropolitana: un estudio cualitativo. Rev Med Chile. 2015;143:424-32.

29. Matthey S, Barnett B, Kavanagh DJ, Howie PV. Validation of the Edinburgh Postnatal Depression Scale for men, and comparison of item endorsement with their partners. J Affect Disord. 2001;64(23):175-84.

30. Areias ME, Kumar R, Barros H, Figueiredo E. Comparative incidence of depression in women and men, during pregnancy and after childbirth. Validation of the Edinburgh Postnatal Depression Scale in Portuguese mothers. Br J Psychiatry. 1996;169(1):30-5.

31. Condon J, Boyce P, Corkindale C. The First-Time Fathers Study: a prospective study of the mental health and wellbeing of men during the transition to parenthood. . Aust N Z J Psychiatry. 2004;38(1-2):56-64.

32. Veskrna L. Peripartum depression-does it occur in fathers and does it matter? J Mens Health. 2013;7(4):420-30.

33. Yogman M, Garfield CF, Bauer NS, et al. Fathers' Roles in the Care and Development of Their Children: The Role of Pediatricians. Pediatrics. 2016;138(1). 